\title{
Immunogenical Study of Chimeric Recombinant Intimin-Tir of Escherichia coli O157:H7 in Mice
}

\author{
Alavieh Yazdanparast ${ }^{1}$, Seyed Latif Mousavi ${ }^{1, *}$, Iraj Rasooli ${ }^{1}$, Jafar Amani ${ }^{2}$, Mohammadreza \\ Jalalinadoushan $^{3}$ \\ 1 Department of Biology, Faculty of Basic Sciences, Shahed University, Tehran, IR Iran \\ ${ }_{3}^{2}$ Applied Microbiology Research Center, Baqiyatallah University of Medical Sciences, Tehran, IR Iran \\ 3 Department of Pathology, School of Medicine, Shahed University, Tehran, IR Iran \\ ${ }^{*}$ Corresponding author: Seyed Latif Mousavi, Department of Biology, Faculty of Basic Sciences, Shahed University, Tehran, IR Iran. Tel.: +98-2151212200, Fax: +98-2151212201, E-mail: \\ slmousavi@shahed.ac.ir.
}

Received: February 19, 2012; Revised: February 25, 2012; Accepted: March 06, 2012

\begin{abstract}
Background: Enterohemorrhagic Escherichia coli (EHEC) O157:H7 causes hemorrhagic colitis. The most important reservoirs of this bacterium are domestic ruminant, particularly the cattle. Although it has low prevalence, the severity of consequent diseases of this bacterium brings concerns to develop effective vaccines. This bacterium is able to produce attaching-effacing (A/E) lesions in intestinal epithelial cells and several genes have been implicated in $\mathrm{A} / \mathrm{E}$ formation. EspA is part of the type III secretion systems that could deliver Tir (translocatedintimin receptor) to the host epithelial cell.

Objectives: The eae gene encoded intimin protein which is essential for colonization of the mucosa and A/E lesions formation by docking to the Tir.

Materials and Methods: Based on these concepts, the immunogenic part of eae and tir genes were constructed and fused together by a linker. The synthetic constructs were subcloned on pET28a + vector and expressed in E. coli BL21DE3. The purified chimeric recombinant protein was injected to mice and the rising antibody was assessed.

Results: The results showed that the chimeric recombinant protein induced strong humoral response as well as protection against live oral challenges using E. coli O157:H7.

Conclusions: The rIT could reduce bacterial shedding effectively.

Keywords: Escherichia coli O157:H7; Tir protein, E. coli
\end{abstract}

\section{Introduction}

Enterohemorrhagic Escherichia coli (EHEC) constitute an important group of zoonotic enteric pathogens including EHEC O157:H7 which is the most common cause of bloody diarrhea or hemorrhagic colitis in the world (1). Cattles are the primary source and key control points of EHEC. Contamination by this organism is commonly acquired in humans through contact with ruminant feces $(2,3)$. Human infection may cause acute gastroenteritis and in some cases is followed by hemolytic uremic syndrome (HUS) and hemorrhagic colitis with severe renal and neurological sequelae (4). Furthermore, EHEC O157:H7 like other Shiga-like toxin producing E. coli (STECs) can be life threatening via producing shiga toxins $(5,6)$. EHEC belongs to a family of pathogens that cause attaching and effacing lesions (7) and develops attaching and effacing (A/E) lesions on mammalian cell in vitro and in vivo $(8,9)$. Like other diarrhea producing (entropathogenic, EPEC) E. coli, this intestinal pathogen seems to perturbs the intestinal barrier involving degeneration of epithelial brush border microvilli and the formation of actin-rich pedestals within the host cell beneath the adherent bacteria (10). A number of factors include flagella, fimbreae/pili, autotransporters, and a type III secretion system has been identified, contributing EHEC O157 for colonization of gastrointestinal epithelium $(2,11)$.

Attachment to the host cell is mediated primarily by a bacterial outer membrane protein called intimin, encoded by eae gene, in concert with firstly host- considered receptor (12). The carboxy-terminal portion of intimin binds the bacterium-encoded (translocated to host cell membrane) intimin receptor (Tir), resulting in intimate attachment of bacteria to the eukaryotic cell surface (1,

Implication for health policy/practice/research/medical education:

Enterohemorrhagic Escherichia coli (EHEC) are important groups of zoonotic enteric pathogens, including EHEC O157:H7. It is the most common cause of hemorrhagic colitis which can be life threatening by producing shiga toxins. Initial attachment and colonization of EHEC are due to outer membrane proteins like intimin, EspA and secreted proteins such as Tir. Blocking these factors can prevent bacterial binding at early stages. Exposure of the individuals to a recombinant protein involving functional epitopes of these factors could lead to stimulation of antibodies production against bacterial attachment.

Copyright (C) 2012, Shahid Beheshti University of Medical Sciencces; This is an Open Access article distributed under the terms of the Creative Commons Attribution License (http://creativecommons.org/licenses/by/3.0), which permits unrestricted use, distribution, and reproduction in any medium, provided the original work is properly cited. 
13). Sequence analysis of intimins from several species has revealed homology in amino-termini but less identity at their carboxy terminal. Unlike EPEC in which the tyrosine is being phosphorylated in EHEC, the Tir is not tyrosine phosphorylated within the host cell membrane (10). Secretion of Tir is mediated by type III secretion system that delivers these virulence factors into the epithelial cells. The translocation tool assisting in secreting and/or delivery of type three secretion system (TTSS or T3SS) components and implied proteins, is a large filamentous organelle that is transiently present on the bacterial surface and interacts with the host cell during the early stage of A/E lesion formation $(14,15)$. The major component of this filamentous organelle is a structural protein named EspA which mediates binding bacteria to the host cell (13) as well as to translocation of EspB, EspD and Tir into host cells. Following bacterial adhesion to host cell, intimin-Tir interaction causes delivery of virulence factors. This complex process may also result in morphological and functional alterations, including rearrangement of cytoskeleton and modulates signal transduction pathways (10). It is worth taking required measures for a new and efficient vaccine against this organism, since not only using antibiotics causes enhanced pathogenesis (16) but also it is less efficient comparing to the current therapeutic methods (17).

It has been the long-term goals of scientific parties to develop an effective vaccine in ordr to prevent cattle from infection and consequent transmission of EHEC O157:H7 to humans $(18,19)$. Limiting colonization and shedding of EHEC 0157:H7 from terminal rectum of the cattle would decrease human exposure to this pathogen. The organism causes limited pathology at terminal rectum, hence it is most likely that the vaccine work by simply blocking bacterial adherence (2). As mentioned above, the factors contributing to EHEC O157:H7 initial attachment and colonization are outer membrane proteins like intimin, EspA and secreted proteins such as Tir. Blocking these factors can lead to the prevention of bacterial binding at very early stages. It was hypothesized that a recombinant protein, involving functional epitopes of these factors lead to the development of multimeric protein chimer. The resultant has the ability to stimulate antibodies which award drastic resistance to bacterial attachment.

\section{Objectives}

The aim of this study was to analyze the effect of the divalent chimeric protein composed of intimin and Tir (rIT) in prevention of the mice rectal epithelium colonization by EHEC 0157:H7 and subsequent reduction of shedding. The interactive epitops of the mentioned proteins are selected according to their intimate role in bacterial attachment. Finally, the effect is compared with previous studies.

\section{Materials and Methods}

\subsection{Amplification of Synthetic Gene}

The divalent fragment including 282 amino acids from C-terminal of intimin (intimin 282, the part which interacts with Tir) and 103 amino acids of Tir (residues 258-361 which interact with intimin), were amplified via PCR from a previously described trivalent synthetic gene, GenBank accession number FJ744505 (20). The primers used included 5'-ATCTATGAATTCTTTGATCAGACCAAAGCG-3' as forward eae, and 5'-ATCTACTAAGCTTTTAGCCCGCGCCGC-3' as reverse tir. The restriction sites EcoRI and HindIII were inserted at the $5^{\prime}$ end of primers respectively.

\subsection{Cloning, Expression and Purification of Recom- binant Chimeric Proteins}

The PCR amplified Intimin-Tir (IT) fragment digested with respect to enzymes and were cloned into pET28a. The recombinant plasmid was transformed to E. coli BL21 DE3 calcium treated competent cells in heat-cold shock procedure. Screening of true clones was examined by digestion and PCR. Recombinant E. coli BL21 DE3 contained IT fragment were cultured until OD600nm: 0.6, then induced with $1 \mathrm{mM}$ IPTG for laboratory scale production. Recombinant divalent proteins were expressed and the cells were harvested by centrifugation at $5000 \mathrm{rpm}$ for 5 min. Purification was done by Ni-NTA column (QIAGEN) under native condition and verified by SDS-PAGE followed by coomassie blue staining. Protein concentration was assessed via Bradford assay.

\subsection{Western Blotting}

Western blotting was analyzed with specific anti $6 \times$ His tag antibody for both recombinant chimer proteins. Induced and un-induced recombinants E. coli BL21 DE3 were selected as test and control groups, respectively. The clear cell lysate was run on SDS-PAGE and were blotted onto nitrocellulose membrane. The membrane was blocked using 5\% skim milk in PBS. After 3 times of washing, using PBS/T (PBS containing 0.05\% Tween 20), 1:8000 dilution of anti-histidine was applied on membrane for $45 \mathrm{~min}$ in $37^{\circ} \mathrm{C}$. After washing, the substrate was added and reaction was stopped by distilled water.

\subsection{Animal Immunization With Recombinant Pro- teins}

Two groups of BALBC/c (Pasteur Institute of Iran) were immunized. Test group was administrated subcutaneously with chimeric protein along with complete Freund's adjuvant (SIGMA) (Table 1). 
The control group received adjuvant with sterile PBS. Boosting was performed 14 and 28 days later with in- complete Freund's adjuvant, and blood samples were obtained after each boosting from mice eyes.

Table 1. Summary of Immunization Protocols

\begin{tabular}{lllllll}
\hline \multirow{2}{*}{ Groups } & \multicolumn{2}{c}{ Initial Immunization } & \multicolumn{2}{c}{ Booster $\mathbf{1}$} & \multicolumn{2}{c}{ Booster 2 } \\
\cline { 2 - 7 } & Components & Quantity $(\mu \mathbf{L})$ & Components & Quantity $(\mu \mathbf{L})$ & Components & Quantity $(\mu \mathbf{L})$ \\
\hline Control, $(\mathbf{n}=\mathbf{4})$ & PBS & 100 & PBS & 100 & PBS & 100 \\
& C.F.A & 100 & I.F.A & 100 & I.F.A & 100 \\
rIT, $(\mathbf{n}=\mathbf{4})$ & rIT & $(5 \mu \mathrm{g})$ & IIT & $(5 \mu \mathrm{g})$ & rIT & $(5 \mu \mathrm{g})$ \\
& C.F.A & 100 & I.F.A & 100 & I.F.A & 100 \\
\hline
\end{tabular}

a Abbreviations: C.F.A, complete Freund's adjuvant; I.F.A, incomplete Freund's adjuvant

\subsection{Quantification of Specific Humoral Antibody}

ELISA (enzyme-linked immunosorbent assay) was used to quantify antibody rise in theserum of control and immunized mice. The ELISA plate were coated overnight at $4^{\circ} \mathrm{C}$ with $5 \mu \mathrm{g}$ of rIT diluted in $100 \mu \mathrm{L}$ carbonate buffer (0.05 M, pH = 9.6). After each step, wells were washed with PBS/T (0.05\% Tween 20). Non-specific binding sites were blocked with $100 \mu \mathrm{L}$ of $5 \%$ dry milk in PBS. Serial dilutions for each test and control group serums were prepared beginning from 1/200 in PBS (100 $\mu \mathrm{L}$ per well) and incubated for one hour in $37^{\circ} \mathrm{C}$. After washing with PBS/T, antimouse IgG conjugate (SIGMA) 1/5000 diluted in PBS was added. Following final washing, color was developed by addition of OPD as a substrate and reaction was stopped with $\mathrm{H} 2 \mathrm{SO} 4$ 2M. The OD492 nm was measured using microplate reader (PERLONG).

\subsection{Characterization of Antibodies Against Chi- mera Protein}

ELISA was applied to assess Ag-Ab and Ab-whole cell (of bacteria) interaction level. The purified chimer divalent protein (rIT) was assayed with antibodies raised against trivalent chimer protein consisting of EspA120Intimin282-Tir103 obtained from our previous work (16). Serum of immunized mice raised against rIT was also assayed with the trivalent protein (rEIT).

\subsection{Live Assay}

Fourteen days after final administration, the mice were treated with water containing $5 \mathrm{mg} / \mathrm{mL}$ streptomycin overnight. The mice were challanged orally with 1010 CFU E. coli O157:H7 from reference laboratory of Booali Hospital (ATCC: 35218 containing espA, eae, tir genes), suspended in $100 \mu \mathrm{L}$ sterile PBS per mouse. CFU/gr faces of bacteria were enumerated alternate days in 14 days by plating $100 \mu \mathrm{L}$ of 1:10 w/v dilution of feces in LB broth onto sorbitol MacConkey agar containing $0.5 \mathrm{mg} / \mathrm{l}$ cefexime and $2.5 \mathrm{mg} / \mathrm{l}$ tellurite to differentiate other E. coli strains other than 0157:H7. Serial dilution of feces was continued to have no more growth on plate and an average of enumeration of several dilutions were calculated and reported as total bacterial shedding for each mouse.

\subsection{Tissue Sampling}

Three weeks after challenge, the mice were sacrificed and colon and cecum were collected for histological analysis. Each biopsy was fixed in 10\% formaldehyde and sent to pathology assay. Tissue sections were stained with hematoxylin and eosin and checked by microscope.

\subsection{Analytical Comparison}

The data in each figure was a representative of three experiments expressed as the mean \pm standard deviation (SD). All statistical analyses were done by SPSS (ver. 15.0) statistical program. ANOVA test was used to compare antibody responses between immunized and control groups. Evaluation and diagnosis of differences in EHEC colonization inhibition on intestinal epithelium surface of the mice were also performed with ANOVA test. A P value of less than 0.05 was considered statistically significant.

\section{Results}

\subsection{Subcloning of Chimera Gene}

Primers were applied for subcloning of fragment in E. coli BL21DE3 on expression vector pET28a. Fragment proliferated by PCR is displayed in Figure $1 \mathrm{~A}$. The cloned chimer recombinants digested with related restriction enzymes are shown in Figure $1 \mathrm{~B}$.

\subsection{Expression and Purification of Chimera Pro- tein}

Recombinant IT protein was expressed with N-terminal His-tag and examined by SDS-PAGE and coomassie blue staining. Molecular weight of recombinant proteins was identified as anticipated. The SDS-PAGE analysis of the purified proteins is shown in Figure $2 \mathrm{~A}$. The $46 \mathrm{KD}$ bands 
belong to rIT protein according to their expected molecular weight. The expression was confirmed by anti-His antibodies using western blotting (Figure 2 B).

Figure 1. PCR Products and Digestion Analysis of Some Accidental Selected Transformed Clones on Agarose Gel 1\%

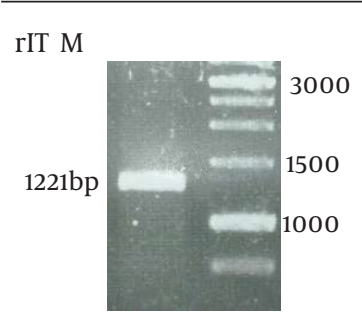

(A)

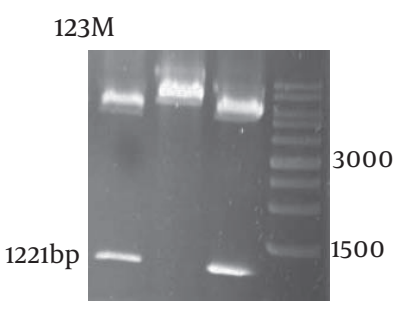

(B)

A) Lane M, DNA size marker. Lane rIT, recombinant Intimin-Tir. B) Lane M, DNA size marker $1 \mathrm{~kb}$. Lane 1-3, digested construct of rIT

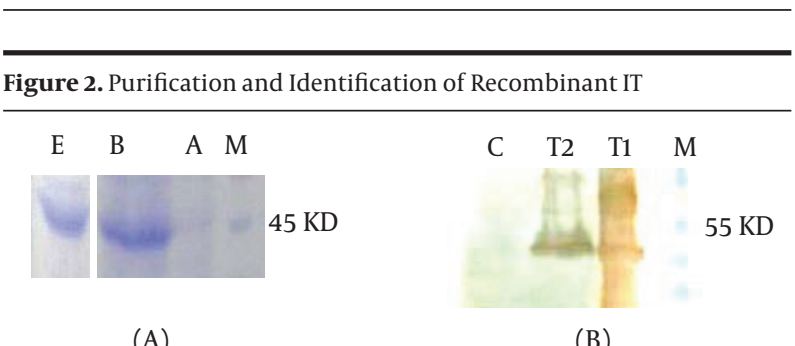

(A)

(B)
A) Purification steps of recombinant IT, M, protein size marker. B, cleared lysate before column. A, cleared lysate after column. E, elution with 250 mM imidazole. B) Western blotting of recombinant IT. M, protein size marker. C, non-induced transformed BL21DE3 as control. T1, supernatant of induces transformed BL21DE3. T2, purified chimeric recombinant protein.

Protein purification was done in native condition. Elutions (250 mM imidazole), containing protein concentration about $0.3 \mu \mathrm{g} / \mu \mathrm{L}$ which were determined by Bradford protein assay.

\subsection{Serum Antigen Specific Responses}

High titers of antibody response in immunized mice sera were observed compared to control groups using ELISA method. Raising antibody titer in immunized mice serums appeared significant after the first and second boosters in dilution 1/256,000 (Figure 3).

\subsection{Characterization of Recombinant Antigen}

To confirm purified proteins as the right favourite antigens and recognize their specificity, divalent protein was assayed with the anti-rEIT. In addition, anti-rIT was used to interact with trivalent rEIT. Their interaction was detected and quantified by ELISA assay (Table 2). Unimmunized mice antisera were considered as the control group.
Figure 3. Serum Antibody Response of BALB/C Mice Immunized With Recombinant Protein

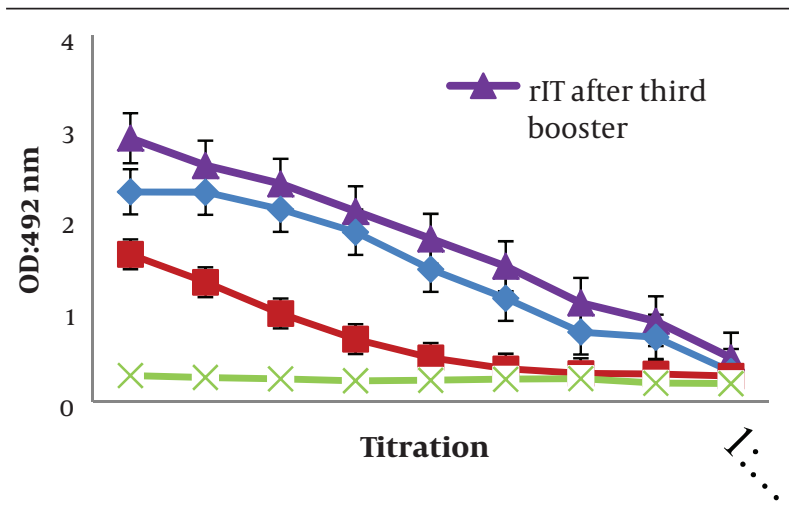

Figure depicts the anti-rit titer in each group.

\begin{tabular}{|c|c|c|}
\hline & Intimin-Tir & EspA-Intimin-Tir \\
\hline Anti Intimin-Tir & $25,000^{a}$ & 80,000 \\
\hline Anti EspA-Intimin-Tir & 80,000 & 120,000 \\
\hline
\end{tabular}

\subsection{Challenging With E. coli 0157:H7 and Compar- ing Bacterial Colonization}

Immunized and unimmunized mice were challanged orally with live E. coli $\mathrm{O} 157$ :H7 to deduce the ability of antibodies to prevent colonization of bacteria on the epithelium mice intestine. The shedding results demonstrated that anti-rIT effectively worked in prevention of attachment and colonization of bacteria (Figure 4).

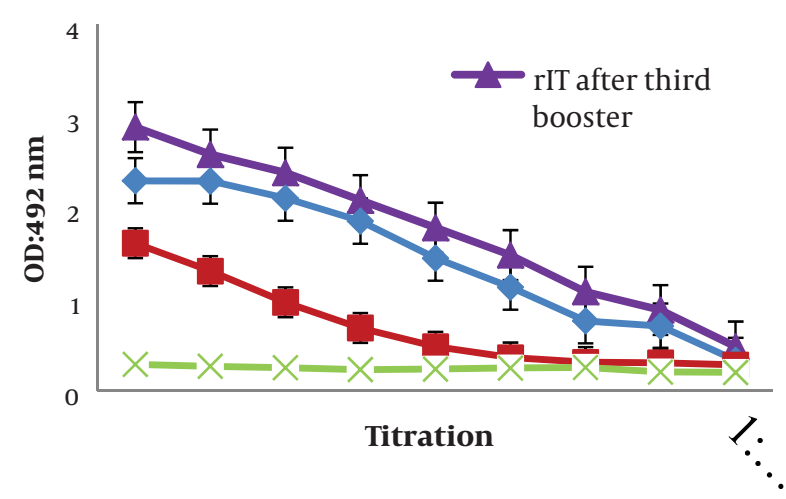

Figure 4. E. coli $\mathrm{O} 157$ :H7 shedding in feces following subcutaneous administration in mice. Mice were immunized with rIT protein. Two weeks following the secondary immunization, the mice were orally fed 1010 E. coli O157:H7 and shedding was monitored in the feces for 14 days. 
The gradient of decrease in EHEC Log10CFU/gr in test groups compared to control in 2 weeks shows different rates of shedding (Figure 4 ).

\subsection{Tissue Assay}

Tissue sections of distal intestine from the mice which received PBS/adjuvant (as control) and rIT/PBS/adjuvant in two turns which had sent for pathology surveys showed that rIT reduced the volume of attaching bacteria to the intestinal epithelium and efface the tissue less comparing with the control mice intestinal tissue which was not protected by any vaccine or other preventing factor. Figure 5 illustrates the weakened attaching/effacing effects on epithelial cells and reduced number of bacteria to the epithelium.

Figure 5. Tissue Sampling of Colon and Cecum From Unimmunized and Immunized Infected Mice

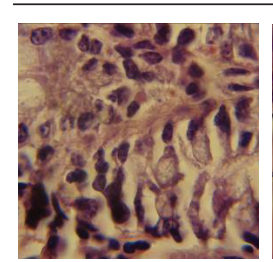

(A)

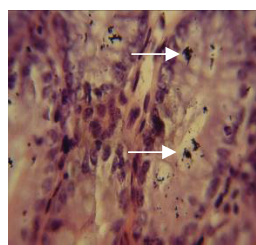

(B)

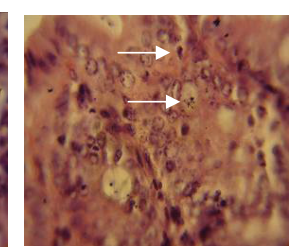

(C)
A) Untreated mice B) Unimmunized infected mice. C) Immunized rIT infected mice. The numbers of bacteria have reduced in $C$ as consequent of Immunized rIT.

\section{Discussion}

Several proteins, especially those encoded by LEE genes have been suggested and investigated as a vaccine candidate to protect cattle against EHEC. Some of these proteins include whole bacterial lysate, purified native virulence proteins, recombinant virulence proteins and finally truncated recombinant proteins from EHEC O157:H7 (2, 3, 16-19, 21-25). In 1998, it was believed that EspA is a major structural protein of filamentous organelle acting as a part of translocating apparatus of TTSS in enterobacteriaceae $(15,26)$ which secretes a number of intestinal virulence factors such as intimin (which docks intimately bacterial cell, onto Tir (bacterial transferred intimin receptor) (27). It also elucidated by Dean et al. that EHEC strains require intimin for colonization, $\mathrm{A} / \mathrm{E}$ lesions, and disease in cattle and piglets per infection of newborn calves with intimin-positive or intimin-negative EHEC 0157: H7 (28)). It could be proposed that blocking of biological function of these specific components mediated by their antagonist antibody could impair stability of bacteria on the epitheliual surface. Vilte DA et al. (2008) affirm that bovine colostrum contains immunoglobulin $\mathrm{G}$ antibodies against intimin, EspA, and EspB and could inhibit hemolytic activity mediated by the Type Three Secretion System of A/E lesion producing Escherichia coli in newborn calves (29)

Several studies on purified or recombinant EspA, intimin and Tir proteins, have proved immunogenicity of these proteins $(14,16,24,25)$. Previous studies by Amani et al. on mouse model was carried out with the construct containing EspA, intimin and Tir. In this study, we analyzed the dimeric protein contain intimin and Tir. The effective immunity of intimin has been proved that this protein is more efficient vaccine than other virulence factors (3). This can be the reason why we included this protein in both constructs. On the other hand, the EspA protein acts as an important factor in attachment of bacteria and if it is blocked, the Tir protein will not be delivered and consequently the attachment of bacteria will be demolished (18). These arguments may be convincing enough to employ an approach for designing antigen/ antigens, considered the main protein candidate studied yet. Using multiepitope antigens is one of the recent methods of increasing immunogenicity of the antigens. Meanwhile using chimeric antigens can help us for better understanding and characterization of each individual protein participating in the chimer.

In this study, we demonstrated that subcutaneous vaccination of mice with recombinant chimera protein including Intimin282-Tir103 can develop high titer of IgG in the serum of immunized mice. Comparing ELISA results of this divalent chimera protein with the trivalent chimera protein (16) indicates that the trivalent chimeric antigen can stimulate humoral responses more strongly. ELISA results revealed higher titer for Intimin-Tir. It supports the results obtained from live challenges with bacteria. The unimmunized control mice did not show a significant raise in antibody titer. The specific antibody against divalent protein (IT) could be able to recognize the native intimin in the whole cell and secreted protein of EHEC bacteria (Data not shown). It has been demonstrated that selected portions of proteins could stimulate humoral antibodies which could recognize native intimin and Tir. McNeilly et al. (2010) demonstrated that systematic vaccination of cattle with a combination of purified intimin531, EspA and Tir significantly reduces shedding of E. coli O157:H7 following oral challenge. It is worth mentioning thatimmunizing animals via these methods individually produce serological responses, however it does not protect animals from oral challenges (24).

Statistical analysis showed significance differences between test and control groups for both cases of ELISA and live assay results. Subsequently the results of vaccination of rIT confirm inhibition of intestinal colonization of $E$. coli $\mathrm{O} 157: \mathrm{H} 7$ in mice Results of live assay showed the rIT could prevent EHEC 0157:H7 from attaching to the epithelium and shorten the duration of fecal bacteria shedding. According to the observations (Figure 4) rIT was able to prevent colonization of the bacteria in the intestine as confirmed by bacterial shedding in feces of the test group decreased with a significant difference compared 
to that of control group during 2 weeks monitoring. Our study showed that Intimin-Tir (IT) recombinant protein can be considered as candidate vaccines and, which has the ability to prevent EHEC attachment to and colonization in the intestinal epithelium of the mice.

\section{Acknowledgements}

We wish to thank our laboratory in charge Ms. Shakiba, Darvish, Alipour, Astaneh for providing technical assistance to conduct this work.

\section{Authors' Contribution}

The core idea of this work was laid down by Prof. S. L. Mousavi who has also collected the data and edited this manuscript jointly with Prof. Iraj Rasooli. Mrs. Alavieh Yazdanparast, an M.Sc student of Shahed University conducted the project as a part of her dissertation. Dr. Jafar Amani acted as technical advisor. Prof. Mohammadreza Jalalinadoushan contributed to pathological studies of the present work.

\section{Financial Disclosure}

The authors declare that they have no competing interest.

\section{Funding/Support}

The study is supported by Shahed University and Biotechnology Development Council of I. R. Iran, Basic Sciences Research Centre with grant No. 57243.

\section{References}

1. Judge NA, Mason HS, O'Brien AD. Plant cell-based intimin vaccine given orally to mice primed with intimin reduces time of Escherichia coli O157:H7 shedding in feces. Infect Immun. 2004;72(1):16875.

2. McNeilly TN, Mitchell MC, Rosser T, McAteer S, Low JC, Smith DG, et al. Immunization of cattle with a combination of purified intimin-531, EspA and Tir significantly reduces shedding of Escherichia coli $\mathrm{O} 157: \mathrm{H7}$ following oral challenge. Vaccine. 2010;28(5):14228.

3. van Diemen PM, Dziva F, Abu-Median A, Wallis TS, van den Bosch $H$, Dougan G, et al. Subunit vaccines based on intimin and Efa1 polypeptides induce humoral immunity in cattle but do not protect against intestinal colonisation by enterohaemorrhagic Escherichia coli $\mathrm{O} 157: \mathrm{H} 7$ or $\mathrm{O} 26: \mathrm{H}$. Vet Immunol Immunopathol. 2007;116(1-2):47-58.

4. Wong CS, Jelacic S, Habeeb RL, Watkins SL, Tarr PI. The risk of the hemolytic-uremic syndrome after antibiotic treatment of Escherichia coli O157:H7 infections. N Engl J Med.2000;342(26):1930-6.

5. Donohue-Rolfe A, Kondova I, Mukherjee J, Chios K, Hutto D, Tzipori S. Antibody-based protection of gnotobiotic piglets infected with Escherichia coli $0157: \mathrm{H} 7$ against systemic complications associated with Shiga toxin 2. Infect Immun. 1999;67(7):3645-8.

6. Tarr PI, Gordon CA, Chandler WL. Shiga-toxin-producing Escherichia coli and haemolytic uraemic syndrome. The Lancet. 2005;365(9464):1073-1086.

7. Phillips AD, Navabpour S, Hicks S, Dougan G, Wallis T, Frankel G. Enterohaemorrhagic Escherichia coli O157:H7 target Peyer's patches in humans and cause attaching/effacing lesions in both human and bovine intestine. Gut. 2000;47(3):377-81.
8. Knutton S, Baldwin T, Williams PH, McNeish AS. Actin accumulation at sites of bacterial adhesion to tissue culture cells: basis of a new diagnostic test for enteropathogenic and enterohemorrhagic Escherichia coli. Infect Immun.1989;57(4):1290-8.

9. Adu-Bobie J, Frankel G, Bain C, Goncalves AG, Trabulsi LR, Douce $\mathrm{G}$, et al. Detection of intimins alpha, beta, gamma, and delta, four intimin derivatives expressed by attaching and effacing microbial pathogens. J Clin Microbiol.1998;36(3):662-8.

10. DeVinney R, Stein M, Reinscheid D, Abe A, Ruschkowski S, Finlay BB. Enterohemorrhagic Escherichia coli O157:H7 produces Tir, which is translocated to the host cell membrane but is not tyrosine phosphorylated. Infect Immun. 1999;67(5):2389-98.

11. Yoon JW, Hovde CJ. All blood, no stool: enterohemorrhagic Escherichia coli O157:H7 infection. J Vet Sci. 2008;9(3):219-31.

12. Dean P, Kenny B. Intestinal barrier dysfunction by enteropathogenic Escherichia coli is mediated by two effector molecules and a bacterial surface protein. Mol Microbiol. 2004;54(3):665-75.

13. Hartland EL, Daniell SJ, Delahay RM, Neves BC, Wallis T, Shaw RK, et al. The type III protein translocation system of enteropathogenic Escherichia coli involves EspA-EspB protein interactions. Mol Microbiol. 2000;35(6):1483-92.

14. Kuhne SA, Hawes WS, La Ragione RM, Woodward MJ, Whitelam GC, Gough KC. Isolation of recombinant antibodies against EspA and intimin of Escherichia coli O157:H7. J Clin Microbiol. 2004;42(7):2966-76.

15. Frankel G, Phillips AD, Rosenshine I, Dougan G, Kaper JB, Knutton S. Enteropathogenic and enterohaemorrhagic Escherichia coli: more subversive elements. Mol Microbiol. 1998;30(5):911-21.

16. Amani J, Salmanian AH, Rafati S, Mousavi SL. Immunogenic properties of chimeric protein from espA, eae and tir genes of Escherichia coli O157:H7. Vaccine. 2010;28(42):6923-9.

17. Vilte DA, Larzabal M, Garbaccio S, Gammella M, Rabinovitz BC Elizondo AM, et al. Reduced faecal shedding of Escherichia coli O157:H7 in cattle following systemic vaccination with gammaintimin C(2)(8)(0) and EspB proteins. Vaccine. 2011;29(23):3962-8.

18. Dean-Nystrom EA, Gansheroff LJ, Mills M, Moon HW, O'Brien AD Vaccination of pregnant dams with intimin(O157) protects suckling piglets from Escherichia coli O157:H7 infection. Infect Immun. 2002;70(5):2414-8.

19. Horne C, Vallance BA, Deng W, Finlay BB. Current progress in enteropathogenic and enterohemorrhagic Escherichia coli vaccines. Expert Rev Vaccines. 2002;1(4):483-93.

20. Amani J, Mousavi SL, Rafati S, Salmanian AH. In silico analysis of chimeric espA, eae and tir fragments of Escherichia coli O157:H7 for oral immunogenic applications. Theor Biol Med Model. 2009;6:28.

21. Gyles CL. Shiga toxin-producing Escherichia coli: an overview. J Anim Sci. 2007;85(13 Suppl):E45-62.

22. Yekta MA, Goddeeris BM, Vanrompay D, Cox E. Immunization of sheep with a combination of intimingamma, EspA and EspB decreases Escherichia coli $\mathrm{O} 157: \mathrm{H} 7$ shedding. Vet Immunol Immunopathol. 2011;140(1-2):42-6.

23. Babiuk S, Asper DJ, Rogan D, Mutwiri GK, Potter AA. Subcutaneous and intranasal immunization with type III secreted proteins can prevent colonization and shedding of Escherichia coli O157:H7 in mice. Microb Pathog. 2008;45(1):7-11.

24. Dziva F, Vlisidou I, Crepin VF, Wallis TS, Frankel G, Stevens MP Vaccination of calves with EspA, a key colonisation factor of Escherichia coli $\mathrm{O} 157: \mathrm{H} 7$, induces antigen-specific humoral responses but does not confer protection against intestinal colonisation. Vet Microbiol. 2007;123(1-3):254-61.

25. Stevens MP, van Diemen PM, Dziva F, Jones PW, Wallis TS. Options for the control of enterohaemorrhagic Escherichia coli in ruminants. Microbiology. 2002;148(Pt 12):3767-78.

26. Galan JE, Wolf-Watz H. Protein delivery into eukaryotic cells by type III secretion machines. Nature. 2006;444(7119):567-73.

27. Miyake M, Hanajima M, Matsuzawa T, Kobayashi C, Minami M Abe A, et al. Binding of intimin with Tir on the bacterial surface is prerequisite for the barrier disruption induced by enteropathogenic Escherichia coli. Biochem Biophys Res Commun. 2005;337(3):922-7. 
28. Dean-Nystrom EA, Bosworth BT, Moon HW, O'Brien AD. Escherichia coli $\mathrm{O} 157: \mathrm{H} 7$ requires intimin for enteropathogenicity in calves. Infect Immun. 1998;66(9):4560-3.

29. Vilte DA, Larzabal M, Cataldi AA, Mercado EC. Bovine colostrum contains immunoglobulin $\mathrm{G}$ antibodies against intimin, EspA, and EspB and inhibits hemolytic activity mediated by the type three secretion system of attaching and effacing Escherichia coli. Clin Vaccine Immunol. 2008;15(8):1208-13. 